\title{
Mixed Convection in Lid-Driven "T" Shallow Cavity Heated From Bottom and Filled by Two Immiscible Fluids of Air and $\mathrm{Al}_{2} \mathrm{O}_{3}$-Water Nanofluid
}

\author{
Aimad koulali ${ }^{1, *}$, Bachir Meziani ${ }^{2}$, Djamel Sadaoui ${ }^{3}$, Massinissa Adnani ${ }^{1}$, Adel Sahi ${ }^{1}$ \\ ${ }^{1}$ Laboratoire de Physique Théorique, Faculté des Sciences ET Technologie, Université de Bejaia, Bejaia 06000, Algérie. \\ ${ }^{2}$ Laboratoire de Physique Théorique, Faculté de Science Exacte, Université de Bejaia, Bejaia 06000, Algérie. \\ ${ }^{3}$ Laboratoire de Mécanique Matériaux Et Energétique, Faculté Sciences ET Technologie, Université de Bejaia, 06000, Algérie.
}

\begin{abstract}
This work present numerical simulation results of mixed convection in lid-driven "T" shallow cavity, filled by two immiscible fluids layers of air and Al2O3-water nanofluid. Mixed convection condition is created by the upper wall movement and temperature difference between the alveolus bottom and upper wall. Hydrodynamic and thermal characteristics of the flow have been predicted by solving the NavierStokes and energy equation using finite volume method. Coupling between two fluids layers are achieved using continuity of temperature and velocity at the interface air-nanofluid. Nano-particle volume fraction effect and geometrical shape of alveolus sidewalls (plane shape, concave shape and convex shape) have been chosen as discussed parameters. Analysis of obtained results shows that the heat transfer rate decreased with increasing volume fraction of solid inside the nanofluid layer. In addition, geometrical shape of alveolus sidewalls has a poor effect on flow structure and isotherms distribution in the physical domain.
\end{abstract}

\section{Nomenclature}

\begin{tabular}{|c|c|c|}
\hline QUANTITIES & SYMBOL & $\begin{array}{l}\text { COHEREN } \\
\text { T SI UNIT }\end{array}$ \\
\hline Cinematic viscosity $(\mu / \rho)$ & $v$ & $\mathrm{~m}^{2} / \mathrm{s}$ \\
\hline $\begin{array}{l}\text { Diffusivity, Thermal } \\
\left(=k / \rho c_{p}\right)\end{array}$ & $\alpha$ & $\mathrm{m}^{2} / \mathrm{s}$ \\
\hline $\begin{array}{c}\text { Dimensionless Cartesian } \\
\text { Coordinate }\end{array}$ & $\mathrm{X} ; \mathrm{Y}$ & ---------- \\
\hline Dimensionless Pressure & $\mathrm{P}$ & --------- \\
\hline $\begin{array}{c}\text { Dimensionless } \\
\text { velocity Components }\end{array}$ & $\mathrm{U}, \mathrm{V}$ & - \\
\hline Dimensionless Temperature & $\theta$ & ---------- \\
\hline Dynamic viscosity & $\mu$ & $\begin{array}{c}\text { Pa s }=\mathrm{N} \mathrm{s} / \mathrm{m}^{2} \\
=\mathrm{kg} / \mathrm{m} \mathrm{s}\end{array}$ \\
\hline Gravitational acceleration & $g$ & $\mathrm{~m} \cdot \mathrm{s}^{-2}$ \\
\hline Height & $H$ & $\mathrm{~m}$ \\
\hline Length & $\mathrm{L}$ & $\mathrm{m}$ \\
\hline $\begin{array}{l}\text { Velocity components in } \\
\text { Cartesian coordinates } \mathrm{x}, \mathrm{y}\end{array}$ & $\mathrm{u} ; \mathrm{v}$ & $\mathrm{m} / \mathrm{s}$ \\
\hline Reference Velocity & $\mathrm{u}_{0}$ & $\mathrm{~m} / \mathrm{s}$ \\
\hline Solid volume Fraction & $\varphi$ & ----------- \\
\hline Temperature absolute & $T$ & $\mathrm{~K}$ \\
\hline Thermal expansion & $\beta$ & $\mathrm{K}^{-1}$ \\
\hline Thermal conductivity & $K$ & $\mathrm{~W} \cdot \mathrm{m}^{-1} \cdot \mathrm{K}^{-1}$ \\
\hline \multicolumn{3}{|c|}{ SUBSCRIPTS AND SUPERSCRIPTS } \\
\hline Hot & \multicolumn{2}{|c|}{$\mathrm{h}$} \\
\hline Cold & \multicolumn{2}{|c|}{$\mathrm{c}$} \\
\hline Air-region & \multicolumn{2}{|c|}{$(1)$} \\
\hline $\begin{array}{l}\text { Nanofluid-region or water- } \\
\text { region }\end{array}$ & \multicolumn{2}{|c|}{ (2) } \\
\hline Nanofluid & \multicolumn{2}{|c|}{$\mathrm{nf}$} \\
\hline
\end{tabular}

\section{Introduction}

Mixed convection in the presence of two confined fluids has many industrial applications, we find this phenomenon present in the cooling processes of dualfuel engines, and in cooling processes of nuclear power plants, the building with a specific problem... etc. this phenomenon has been studied by many scientific researches. With different geometric considerations and different boundary conditions. In the horizontal channel with permeable walls, [1] provided an analytical study of the heat transfer problem in the presence of two immiscible fluids with an oscillatory flow. Numerous studies has been developed to discuss certain phenomena that occur in the presence of two immiscible fluids in square or rectangular cavities, which has been taken into account in $[2-5]$. The two fluids can be separated with a rigid wall, which is treated by [6-8].

In recent years, the notion of nanofluid is appeared, with the objective to improve heat transfer in industrials systems. In this context, various numerical research has been conducted. A study carried out by [9], on the conjugate natural convection in a cavity separated into two portions and filled by different nanofluid. Many parameters have been taken; the results of this study showed that the highest values of the volume fractions of nanoparticles increases heat transfer. this conclusion on the presence of solid nanoparticles within base fluid has also been confirmed in a semi-wave channel geometry by [10], at the existence of two layers of fluid. Their investigation is based on the injection of nanoparticles into one of the two fluids, in order to study their impact on the heat transfer process in a channel. In another study on natural convection in a rectangular enclosure

\footnotetext{
${ }^{*}$ Corresponding author: aimad.koulali $@$,yahoo.com
} 


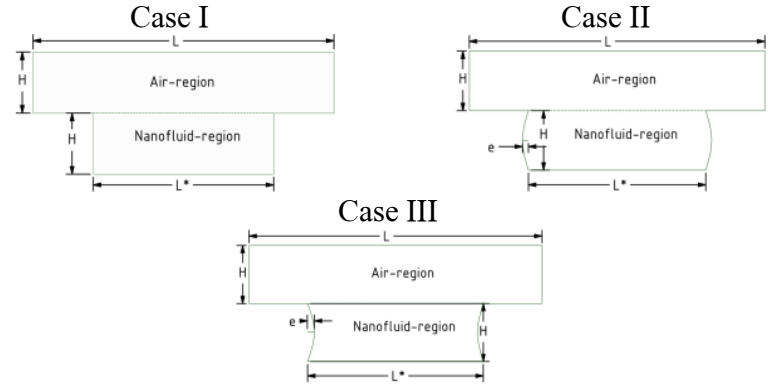

Fig.1. Physical model for the three investigated cases

filled by two immiscible fluids of air and nanofluid, developed by [11], discussion of their results has been focused on the impact of the interface between the two fluids on the structure of the flow pattern in the enclosure.

The impact of adding nanoparticles to base fluid has been widely discussed, with several geometric and boundary conditions considerations. The vast majority of research on the subject indicates that the heat transfer process is positively influenced by the addition of nanoparticles, which has been affirmed by[12-14] in the case of mixed convection, also has been confirmed by [15] in the case of natural convection. However, the results provided by [16] in a numerical study of Rayleigh-Benard natural convection indicates that using a nanofluid can reduce heat transfer, instead than increase it.

The works has been mentioned above led us to have a global vision on the subject. Therefore, it has been found that there is little work dealing with mixed convection in flows of two immiscible fluids with the presence of solid nanoparticles. This article examines mixed convection in ' $\mathrm{T}$ ' shallow cavity in presence of two-layer fluid of air and A12O3-water nanofluid. Vatical thermal gradient is imposed between the top and bottom of cavity. A study was carried out on the effect of solid volume fraction and geometrical shape of sidewalls of the upper part of cavity (alveolus).

\section{Governing equations and problem formulation}

We considered ' $\mathrm{T}$ ' shallow cavity with aspect ratio $(\mathrm{H} / \mathrm{L}=1 / 5)$, filled with air $(\mathrm{Pr}=0.7)$ on the top part and by $\mathrm{A} 12 \mathrm{O} 3$ water nanofluid in the lower part (alveolus). Fig.1 shows the different cases of the geometric model considered. The upper wall of the enclosure is moving in - $\mathrm{x}$ - direction and maintained at a cold temperature Tc. The bottom of the alveolus is exposed at a hot temperature Th. While, other boundaries of enclosure are maintained adiabatic Fig.2. The boundary conditions of temperature and velocity at the air-nanofluid interface were obtained from the stress balance, and the heat flow balance such as those given by [3]. Continuity of velocities and temperature at the interface were shows in Fig.2. The governing equations of the phenomena, is the Navier-Stokes equations and energy equation, for twodimensional laminar flow in steady state. Boussinesq approximation is adopted for the description of density variation of two fluids.

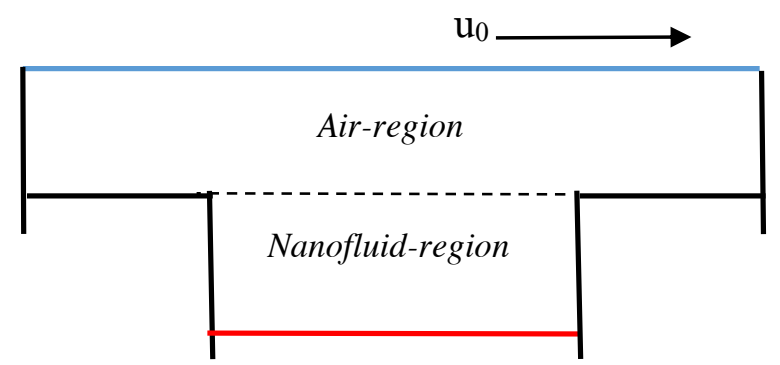

Fig.2. boundary conditions on the physical domain

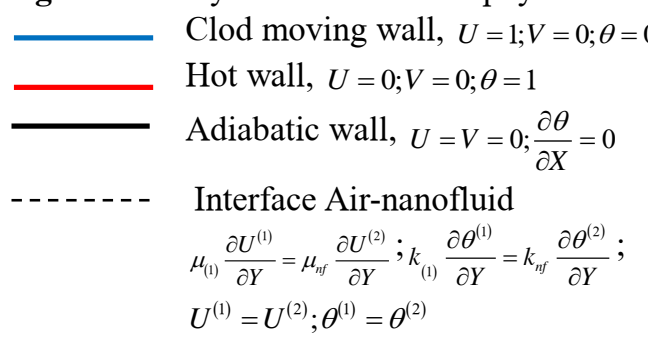

$X=\frac{x}{L} ; Y=\frac{y}{L} ; U^{(1)}=\frac{u^{(1)}}{u_{0}} ; V^{(1)}=\frac{v^{(1)}}{u_{0}} ;$

$P^{(1)}=\frac{p^{(1)}}{\rho^{(1)} * u_{0}^{2}} ; \theta^{(1)}=\frac{T^{(1)}-T_{c}}{T_{h}-T_{c}} ;$

$U^{(2)}=\frac{u^{(2)}}{u_{0}} ; V^{(2)}=\frac{v^{(2)}}{u_{0}} ;$

$P^{(2)}=\frac{p^{(2)}}{\rho^{(2)} * u_{0}^{2}} ; \theta^{(2)}=\frac{T^{(2)}-T_{c}}{T_{h}-T_{c}} ;$

Dimensionless parameters defined in (1), can be used to make the N-S equations and energy equation in dimensionless form. Dimensionless numbers Grashof (Gr), Reynolds (Re) and Prandtl (Pr) are defined as follows:

$$
\begin{gathered}
\operatorname{Re}=\frac{u_{0} L}{v^{(1)}} ; G r=\frac{g \beta^{(1)} \Delta T L^{3}}{\left(v^{(1)}\right)^{2}} \\
\operatorname{Pr}^{(1)}=\frac{v^{(1)}}{\alpha^{(1)}} ; \operatorname{Pr}^{(2)}=\frac{v^{(2)}}{\alpha^{(2)}}
\end{gathered}
$$

Dimensionless form for governing equations is obtained:

For Air-region:

$$
\begin{aligned}
& \frac{\partial U^{(2)}}{\partial X}+\frac{\partial U^{(2)}}{\partial Y}=0 \\
& U^{(1)} \frac{\partial U^{(1)}}{\partial X}+V^{(1)} \frac{\partial U^{(1)}}{\partial Y}=-\frac{\partial P^{(1)}}{\partial X}+\frac{1}{\operatorname{Re}}\left(\frac{\partial^{2} U^{(1)}}{\partial X^{2}}+\frac{\partial^{2} U^{(1)}}{\partial Y^{2}}\right) \\
& U^{(1)} \frac{\partial V^{(1)}}{\partial X}+V^{(1)} \frac{\partial V^{(1)}}{\partial Y}=-\frac{\partial P^{(1)}}{\partial X}+\frac{1}{\operatorname{Re}}\left(\frac{\partial^{2} V^{(1)}}{\partial X^{2}}+\frac{\partial^{2} V^{(1)}}{\partial Y^{2}}\right)+\frac{G r}{\operatorname{Re}^{2}} \theta^{(1)} \\
& U^{(1)} \frac{\partial \theta^{(1)}}{\partial X}+V^{(1)} \frac{\partial \theta^{(1)}}{\partial Y}=\frac{1}{\operatorname{Re}_{P^{(1)}}}\left(\frac{\partial^{2} \theta^{(1)}}{\partial X^{2}}+\frac{\partial^{2} \theta^{(1)}}{\partial Y^{2}}\right)
\end{aligned}
$$

The effective values of thermophysical properties of nanofluid are obtained using the solid volume fraction $\left({ }^{\varphi}\right)$. Where the density of nanofluid is given by:

$\rho_{n f}=(1-\varphi) \rho_{(2)}+\varphi \rho_{p}$ 
The thermal diffusivity of Nanofluid is given as follows:

$$
\alpha_{n f}=\frac{k_{n f}}{(\rho C p)_{n f}}
$$

Knowing that $(\rho C p)_{n f}$ is the effective capacitance for Nanofluid, of which the following expression as proposed by [17]:

$$
(\rho C p)_{n f}=(1-\varphi)(\rho C p)_{(2)}+\varphi(\rho C p)_{p}
$$

Moreover, $(\rho \beta)_{n f}$ which is the specific thermal expansion, is obtained by the following expression:

$$
(\rho \beta)_{n f}=(1-\varphi)(\rho \beta)_{(2)}+\varphi(\rho \beta)_{p}
$$

The effective thermal conductivity of Nanofluid was obtained, using the model form of Maxwell-Garnett's; this form is found to be appropriate by ([18]-[20])

$$
k_{n f}=\left[\frac{\left(k_{p}+2 k_{(2)}\right)-2 \varphi\left(k_{(2)}-k_{p}\right)}{\left(k_{p}+2 k_{(2)}\right)-\varphi\left(k_{(2)}-k_{p}\right)}\right]
$$

In this investigation, we assume the Brinkman model [20], in order to determinate effective dynamic viscosity of Nanofluid, the latter is given by:

$$
\mu_{n f}=\frac{\mu_{(2)}}{(1-\varphi)^{2.5}}
$$

In addition, we introduced the following two reports:

$$
v^{*}=\frac{v^{(1)}}{v^{(2)}} ; \beta^{*}=\frac{\beta^{(1)}}{\beta^{(2)}}
$$

Implementing of Eq. [1.2.8-14], in governing equations, we are obtaining the dimensionless forms as follows:

\section{For Nanofluid-region}

$$
\begin{aligned}
& \frac{\partial U^{(2)}}{\partial X}+\frac{\partial U^{(2)}}{\partial Y}=0 \\
& U^{(2)} \frac{\partial U^{(2)}}{\partial X}+V^{(2)} \frac{\partial U^{(2)}}{\partial Y}=-\frac{\partial P^{(2)}}{\partial X}+\frac{1}{v^{*}} \frac{\rho_{(2)}}{\rho_{n f}} \frac{1}{(1-\varphi)^{2.5}} \frac{1}{\operatorname{Re}}\left(\frac{\partial^{2} U^{(2)}}{\partial X^{2}}+\frac{\partial^{2} U^{(2)}}{\partial Y^{2}}\right)
\end{aligned}
$$

$$
U^{(2)} \frac{\partial V^{(2)}}{\partial X}+V^{(2)} \frac{\partial V^{(2)}}{\partial Y}=-\frac{\partial P^{(2)}}{\partial X}+\frac{1}{V^{*}} \frac{\rho_{(2)}}{\rho_{n f}} \frac{1}{(1-\varphi)^{2.5}} \frac{1}{\operatorname{Re}}\left(\frac{\partial^{2} V^{(2)}}{\partial X^{2}}+\frac{\partial^{2} V^{(2)}}{\partial Y^{2}}\right)
$$$$
+\frac{1}{v^{*} \beta^{*}} \frac{\rho_{(2)}}{\rho_{n f}}\left(1-\varphi+\varphi \frac{\rho_{p} \beta_{p}}{\rho_{(2)} \beta_{(2)}}\right) \frac{G r}{\operatorname{Re}^{2}} \theta^{(2)}
$$

$U^{(2)} \frac{\partial \theta^{(2)}}{\partial X}+V^{(1)} \frac{\partial \theta^{(2)}}{\partial Y}=\frac{1}{V^{*}} \frac{k_{n f}}{k_{(2)}} \frac{(\rho C p)_{(2)}}{(\rho C p)_{n f}} \frac{1}{\operatorname{Re} \operatorname{Pr}^{(2)}}\left(\frac{\partial^{2} \theta^{(2)}}{\partial X^{2}}+\frac{\partial^{2} \theta^{(2)}}{\partial Y^{2}}\right)$

\section{Numerical simulation and code validation}

The governing equations, continuity, momentums and energy equations are integrated on control volumes in order to obtain an algebraic equation system more accessible to the resolution [17]. The SIMPLE algorithm is used to solve the coupled system of algebraic equations. Spatial discretization of the continuity, momentum and energy equations is done by a second order upwind scheme. The interpolation of the pressure is carried out by the PRESTO scheme. To check the convergence of the sequential iterative solution, normalized residual respectively for the continuity, momentum and energy equation is calculated, convergence is obtained when the residual becomes smaller than $10^{-9}$. The numerical code is tested, with comparison isotherms and streamline obtained by this investigation and the results given in [11].

\section{Results and discussion}

In this section, we present the results of the problem simulation. The results were discussed for $\mathrm{Gr}=10^{6}$ and $\mathrm{Re}=1000$, and for three cases according to the geometric shape of the sidewalls of alveolus. The three cases considered are as follows (Plane shape for case I; concave shape for case II, convex shape for case III. Concavity and Convexity degree were chosen from order $\mathrm{e}=0.02 * \mathrm{~L}$.

\subsection{Thermal and flow filed}

\subsubsection{Effect of solid volume fraction $\varphi$}

Initially, we examined the effect of solid volume fraction on the formation of flow patterns within the physical domain studied. Streamline in right of Fig.3, show the appearance of a recirculation zone in the air occupied part, this zone is affected mainly by the mobile cover of cavity. In the lower layer (nanofluid), we notice the formation of two counter-rotating recirculation zones; this observation is mainly due to the heating imposed at alveolus bottom. Here, the nanofluid particles tend to move from alveolus base to air-nanofluid interface. The temperature gradient between the air-nanofluid interface and alveolus bottom create convection regime inside nonfluid particle. In addition, it is interesting to note that an asymmetry of flow occurs in the nanofluid layer, with a large cell to the right of alveolus, and an apparent small cell on the left side. This observation stems from the moving wall direction, where a higher convection rate occurs near the right side of cavity. Solid volume fraction effect on flow pattern are not very significant, where the flow pattern of two contra-rotating cell seems to be dominant for all values of $\varphi$. This effect is exclusively involved in the flow velocity of fluid particles in both layers. Where, according to Fig.3 on the right, it can be seen that streamline density decreases as solid volume fraction value increases. 
Corresponding isotherms are shown in Fig.3 on the left. The first remark that we can see it, is that the imposed boundary condition at the air-nanofluid interface has been represented by continuity of isotherms line at this section of cavity. In addition, from this figure we can see that, the temperature gradient in the lower fluid layers (nanofluid) is poorer by comparing to the temperature gradient inside air-layer. Where, isotherms line density seems to be more pronounced in the upper layer. This difference in temperature distribution inside two fluid layers is mainly due to the difference between fluid properties for each fluid layer. From this, it can be seen that the conductive regime is dominant within the nanofluid-filled part. On the other hand, a purely convective regime is manifested within the air-filled region. As $\varphi$ increases, the temperature gradients within the nanofluid decrease even more. Knowing that it is clear (from Fig.3 on the left) that the isothermal line of “0.98" moves away from the hot wall as $\varphi$ increases. These remarks concerning solid volume fraction effects on flow structure and temperature distribution are valid even for the concave and convex shapes of alveolar side walls (see Fig.4 and Fig.5).

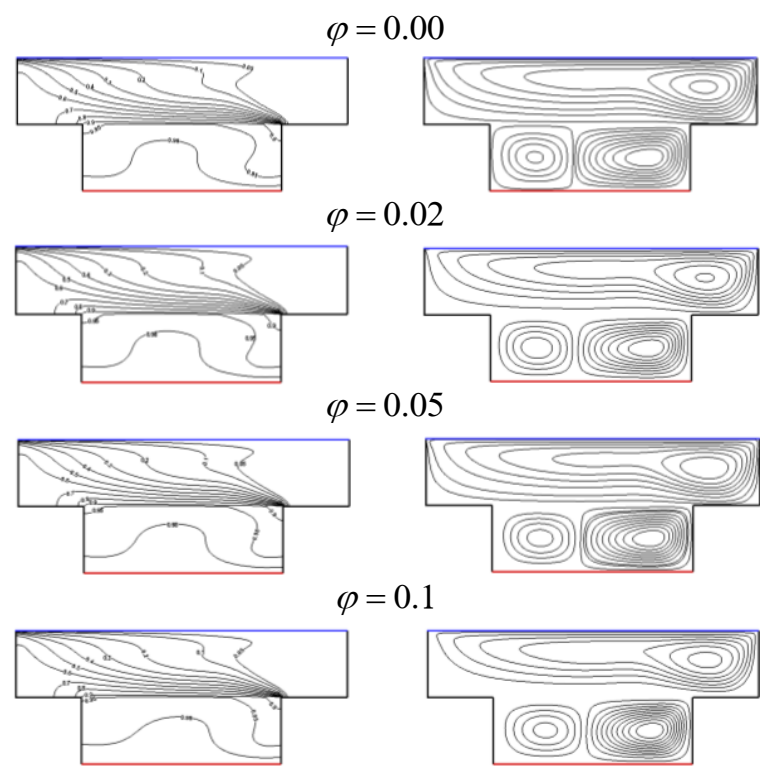

Fig.3. Isotherms (left) and streamline (right), in case I, for different solid volume fractions $\left({ }^{\varphi}\right.$ ) and $\mathrm{Gr}=10^{6}, \mathrm{Re}=1000$.

\subsubsection{Effect of geometrical shape of alveolus sidewalls}

Impact of geometrical shape of alveolus sidewalls on flow structure and temperature distribution can be can be discussed through Fig.3, Fig.4 and Fig.5. Flow structure seem to be similar with both flat and concave shapes (case-I and case-III). This structure was represented by a large cell occupying the entire air layer and two counterrotating cells at nanofluid layer (see Fig.3 on the right and Fig.4 on the right).

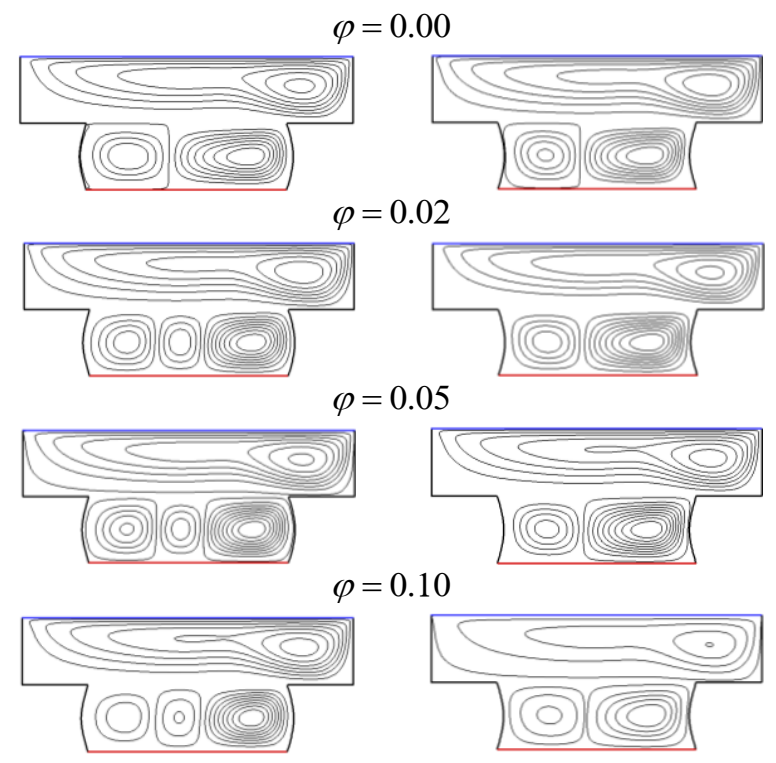

Fig.4. Streamline for two cases of shape-sidewalls of the alveolus (case II in the left and case III in the right), for different solid volume fractions and $\mathrm{Gr}=106, \mathrm{Re}=1000$.

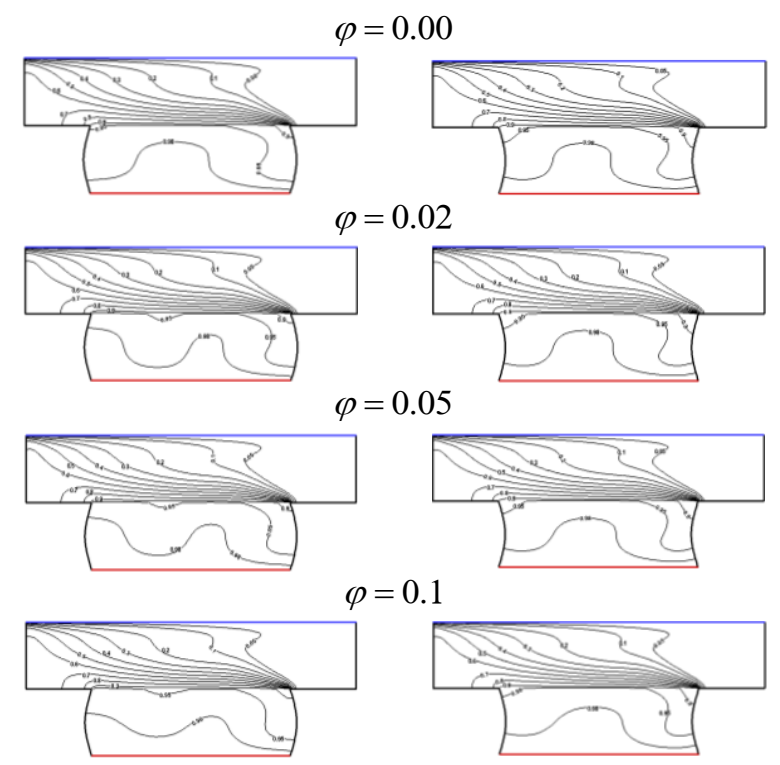

Fig.5. Isotherms for two cases of shape-sidewalls of the alveolus (case I in the left and case II in the right, for different solid volume fractions and $\mathrm{Gr}=106, \mathrm{Re}=1000$.

By comparing stream lines from (Fig.3 on the right and Fig.4), it is clear that with the convex shape and from

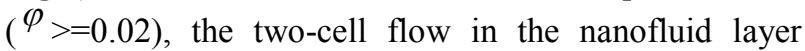
transforms into three-cell flow. Concerning the temperature distribution, comparing the results obtained with the three geometrical shapes of alveolus side walls, it can be seen that the isothermal lines found with a plane shape (case-I) and concave shape (case-III) have the same pattern whatever volume fraction value of solid. However, using the convex shape (case-II) a double thermal plume appears inside the nanofluid layer, this was observed from ( $\varphi=0.02)$ (see Fig.5 on the left). 


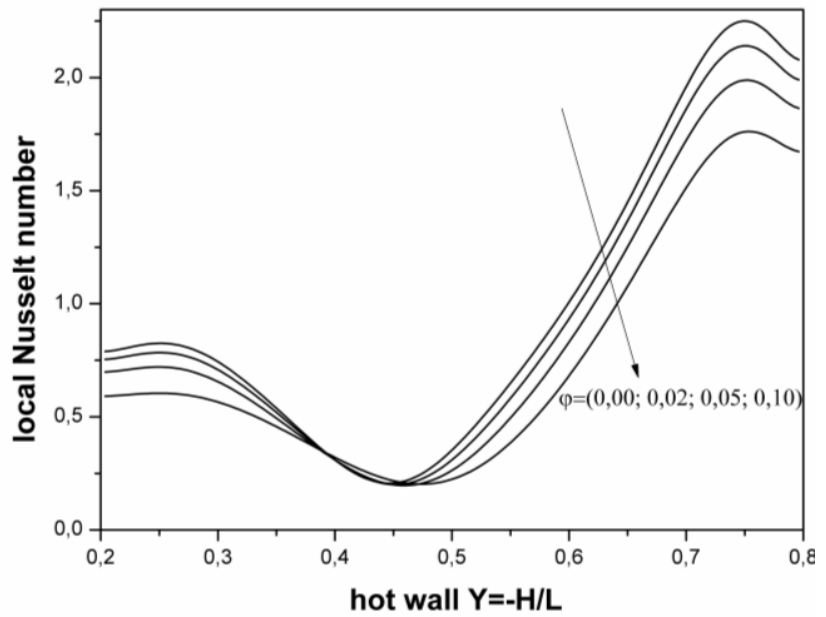

Fig.6. Profile local Nusselt Number at $(\mathrm{Y}=-\mathrm{H} / \mathrm{L})$, for case I with different solid volume fraction of nanofluid.

\subsection{Heat transfer performance}

Heat transfer performance analysis within cavity was performed using local Nusselt evolution at hot wall in case-I (see Fig.6) and average Nusselt number obtained with different geometrical shape cases and various values of solid volume fraction (see Fig.7). According to Fig.6, it was found that the local Nusselt evolution curves followed a similar profile regardless of volume fraction value. It is interesting to note that the local Nusselt values are higher on the right side of hot wall when compared it to left side of wall. Also, minimum values have been recorded at the wall centre, while the peak was observed near to sidewall on the right side. This result confirms the temperature distribution and streamlines presented in previous section. The most important observation that we can draw from Fig.6 is that local Nusselt decreases gradually with increasing solid volume fraction.

Fig.7 illustrates the overall heat transfer rate via the representation of average Nusselt number with the three cases of geometric configuration and various values of volume fraction that are used. From average Nusselt values, it can be concluded that the dominant heat transfer in the nanofluid layer is that of thermal conduction, since average Nusselt values do not exceed 1 in all discussed cases. Also, it is interesting to note that as the solid volume fraction increases, we notice that the average Nusselt number decreases, and this has been observed in all cases of geometrical shape of alveolus sidewalls. In addition, it turned out that the average Nusselt values are the same values with both planar and convex shapes, while the average Nusselt obtained with the concave shape has lower values.

\section{Conclusion}

In this work numerical simulations of the mixed convection flow inside shallow ' $\mathrm{T}$ ' shaped cavity. The cavity was filled with two fluids of air and $\mathrm{Al} 2 \mathrm{O} 3$ waternanofluid. Where air is located in the upper region, while

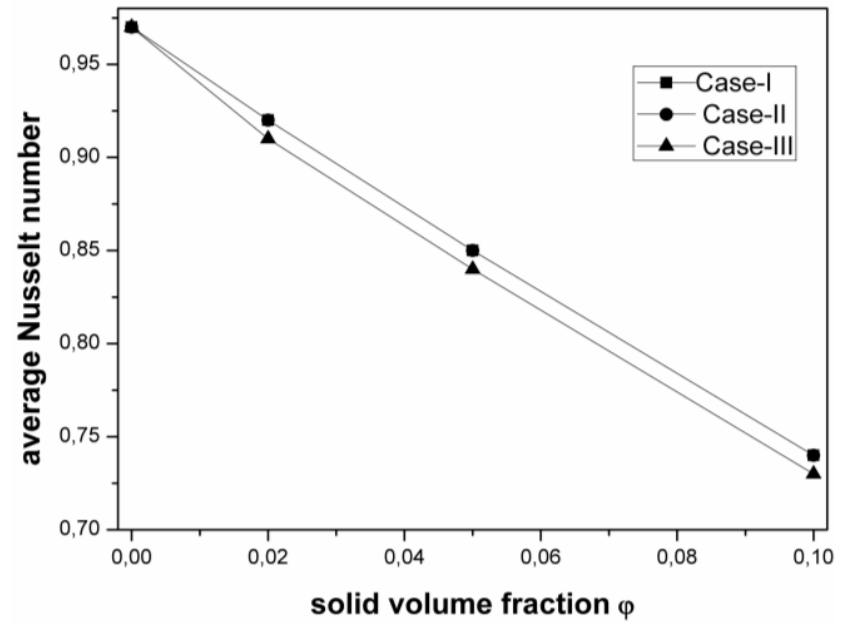

Fig.7. Average Nusselt number as a function of solid volume fraction at hot wall $\mathrm{Y}=-(\mathrm{H} / \mathrm{L})$, for (Case I; Case II; Case III).

the nanofluid layer was placed in lower region (alveolus). The physical model subjected to vertical thermal gradient and the upper wall moving in $\mathrm{x}$ direction. The numerical simulations have been done by fixing $\mathrm{Re}=1000$ and $\mathrm{Gr}=10^{6}$. Effect of solid volume fraction and geometrical shape of alveolus sidewalls have been, considered. Some major results can be expressed as:

- Flow pattern and temperature distribution are affected by geometrical shape of sidewalls of alveolus.

-The plane and convex shape of alveolus sidewall illustrate the same flow structure and the same thermal filed.

- The planar and convex shape guarantee more important heat transfer rate comparing it to concave shape.

-The average Nusselt present decreasing function of solid volume fraction in all discussed cases.

- Under considered thermal and hydrodynamic boundary condition, we have demonstrated that the adding of nanoparticle to base fluid can be reduced the overall heat transfer.

\section{References}

1. A. J. Chamkha, J. C. Umavathi, and A. Mateen, Int. J. Fluid Mech. Res., 31-1,13, (2004).

2. N. P. Moshkin, Int. J. Heat Fluid Flow, 23-3, 373, (2002).

3. J. N. Koster and K. Y. Nguyen, Int. J. Heat Mass Transf., 39-3, 467, (1996).

4. E. H. Malekshah and M. Salari, Exp. Therm. Fluid Sci., 85, 388, (2017).

5. F. S. Oueslati, R. Bennacer, and M. El Ganaoui, Int. J. Therm. Sci., 90, 303, (2015).

6. H. F. Oztop, Y. Varol, and A. Koca, Int. J. Heat Mass Transf., 52-25, 5909, (2009). 
7. A. Koulali, B. Meziani, D. Sadaoui, and M. Adnani, in International Conference on Advanced Mechanics and Renewable Energies ICAMRE2018 November 28 \& 29, 2018 Boumerdes - Algeria, (2018).

8. A. Koulali, B. Meziani, D. Sadaoui, and M. Adnani, SSRN Electron. J., no. November, (2019).

9. F. Selimefendigil and H. F. Öztop, J. Mol. Liq., 216, 67, (2016).

10. Y. Abd Elmaboud, Results Phys., 9, 1643, (2018).

11. M. Salari, E. H. Malekshah, and M. H. Malekshah, Alexandria Eng. J., 57-3, 1401 (2018).

12. M. Rajarathinam, N. Nithyadevi, and A. J. Chamkha, Adv. Powder Technol., 29-3, 590, (2018).

13. R. K. Nayak, S. Bhattacharyya, and I. Pop, Int. J. Heat Mass Transf., 125, 908, (2018).

14. I. Zeghbid and R. Bessaïh, Thermophys. Aeromechanics, 25-2, 245, (2018).

15. Z. Haddad, H. F. Oztop, E. Abu-Nada, and A. Mataoui, Renew. Sustain. Energy Rev., 16-7, 5363, (2012).

16. N. Na, A. Prenosa, T. Nanoteko, P. Ternik, and R. Rudolf, 47-2, 211, (2013).

17. S. V. Patankar, Numerical Heat Transfer and Fluid Flow. (1980).

18. S. Sarkar, S. Ganguly, and A. Dalal, Int. J. Heat Mass Transf., 59-1, 433, (2013).

19. H. Khorasanizadeh, M. Nikfar, and J. Amani, Eur. J. Mech. B/Fluids, 37, 143, (2013).

20. E. Abu-Nada and A. J. Chamkha, Eur. J. Mech. B/Fluids, 29-6, 472, (2010). 